Der Internist informiert regelmäßig alle in Praxis und Klinik tätigen Internisten sowie die internistisch besonders interessierten Praktiker und Allgemeinärzte über alle wichtigen diagnostischen und therapeutischen Entwicklungen im Fachgebiet Innere Medizin.

Das redaktionelle Konzept des Internisten beruht auf folgenden Schwerpunkten:

\section{Wissenschaftliche Kompetenz} und Praxisrelevanz

Fundierte Übersichtsarbeiten renommierter Autoren, ergänzt durch praxisrelevante redaktionelle Ergänzungstexte (,Zum Thema" am Anfang und "Fazit für die Praxis" am Ende jedes Beitrags) bilden den Schwerpunkt der Zeitschrift. Mit diesem Konzept hat sich der Internist seit langem zu einem erstklassigen und unentbehrlichen Medium für die gesamte internistische Fortbildung entwickelt. Die Summe der Einzelthemen bietet dem Leser eine aktuelle Gesamtdarstellung der wichtigsten internistischen Fragestellungen und reflektiert die diagnostischen und therapeutischen Fortschritte des Fachgebietes. Die Übersichtsarbeiten sind in erster Linie auf den praktizierenden Internisten ausgerichtet; wichtige medizinische Randgebiete werden aber auch von Fall zu Fall berücksichtigt.

\section{Strukturierter Weiterbildungsteil (Blaue} Seiten")

Dieser Heftteil bietet dem Assistenzarzt, der sich auf die Fachgebietsanerkennung vorbereitet, komprimierte Informationen zu einer Vielzahl von Krankheitsbildern oder Leitsymptomen. Die Darstellung eignet sich zur schnellen Rekapitulation - auch für den interessierten Arzt in der Praxis. Prüfungserfahrene Fachautoren sind bei der Bearbeitung dieses Heftteils um eine Synthese aus transparenter Textstruktur, Aktualität und Knappheit bemüht.

\section{Einführung zum Thema (Editorial)}

In der Regel kommentiert der jeweilige Heftherausgeber den aktuellen Stellenwert des Heftthemas für den Internisten und leitet daraus Sinn und Zweck der Zusammenstellung der Übersichtsbeiträge und ihrer Standpunkte ab.

4. Die Verlagsredaktion des "Internist“, die mit den Herausgebern eng zusammenarbeitet, koordiniert weitere Rubriken der Zeitschrift:

So berichtet "Medizin aktuell" in erster Linie über mitteilenswerte Fortbildungsveranstaltungen, die - je nach Schwerpunkt - sowoh für den Internisten in der Praxis als auch in der Klinik interessant sind. Das "Kongreßforum" bietet eine Auswahl relevanter nationaler und internationaler Tagungen an.

5. Mitteilungen des BDI ("Gelbe Seiten") Der Berufsverband Deutscher Internisten informiert regelmäßig über wichtige berufs- und verbandspolitische Aspekte. Somit erfährt der Leser alles Wissenswerte über die Positionen und Aktivitäten seines Berufsverbandes.

6. In der Rubrik "Leserbriefe" besteht die Möglichkeit, aktiv in den Dialog mit Herausgebern und Autoren einzutreten. Dieser Diskurs ist wllkommen, weil er zu einer differenzierten Sicht der Dinge beitragen kann und außerdem unterschiedliche Standpunkte und Beurteilungen zuläßt.

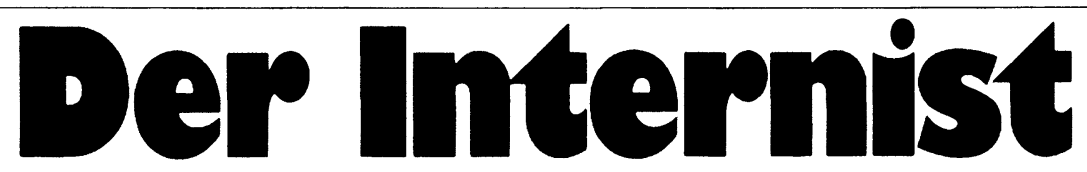

Organ des Berufsverbandes Deutscher Internisten e.V.

\section{Begründet von}

G. Budelmann $\cdot H$. von Kress $\cdot H$. Reinwein

W. Ruge $\cdot H$. Schwiegk . F. Valentin

\section{Unter Mitwirkung von}

H. Weinholz · W. Wildmeister · Hd. Ullmann

(Für den Vorstand des Berufsverbandes Deutscher Internisten e.V.)
R. Aschenbrenner · H.E. Bock · M. Broglie
F. Krück · F. Scheler · R. Schindlbeck
E. Schüller · K. Werdan . E. Wetzels

\section{Herausgegeben von}

M. Classen, München · V. Diehl, Köln

J. van de Loo, Münster - M.P. Manns, Hannover

H.-P. Schuster, Hildesheim .P.C. Scriba, München

W. Siegenthaler, Zürich · B.E. Strauer, Düsseldorf

P. von Wichert, Marburg

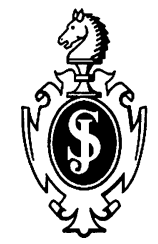

Springer-Verlag Berlin Heidelberg New York London Paris Tokyo Hong Kong Barcelona Budapest 
Band 34 Heft 8 August 1993

Hypogonadismus und Infertilität des Mannes

Redaktion: E. Nieschlag (Münster) und P.C. Scriba (München)

Einführung zum Thema

699

E. Nieschlag, P.C. Scriba

Molekulare Aspekte des Hypogonadismus

T. Gudermann, J. Gromoll

Endokrine und parakrine Regulation der Spermatogenese:

Was ist wichtig für den Arzt?

G.F. Weinbauer, E. Nieschlag

Diagnostik des Hypogonadismus und der Infertilität des Mannes

H.M. Behre, E. Nieschlag

Bildgebende Verfahren bei hypothalamisch-hypophysären

Erkrankungen

J. Schopohl, T. Vogl, C.J. Strasburger

Hypogonadismus und Infertilität als Folge von allgemeinen Erkrankungen und Toxinen

F. Jockenhövel

Therapie des Hypogonadismus und der Infertilität.

Eine kritische Wertung

E. Nieschlag, H.M. Behre

Diagnostik und Therapie der erektilen Dysfunktion

C.G. Stief

Kryokonservierung von Spermien als Zeugungsreserve

für onkologische Patienten

C. Keck, E. Nieschlag

Kasuistik

Redaktion: K. Werdan (München)

Schmerzhafte Hodenschwellung, Dünndarmileus und Fieber

P. Gräf, H. Bierbach, R. Moll

Chronisches Erbrechen und Durchfall nach subtotaler Kolektomie wegen chronischer Obstipation

A. Klauser, B. Wiebecke, L. Schweiberer, S. Müller-Lissner 
Arzneimitteltherapie

Redaktion: F. Scheler (Göttingen)

Azathioprin (Imurek)

P. Berlit, N. Gretz

Mitteilungen des Berufsverbandes Deutscher Internisten

Auf ein Wort

M109

W. Wildmeister

M110

KBV - Vertreterversammlung Dresden 1993

W. Schorre

M115

Eigenverantwortung, Subsidiarität und Solidarität

K. Vilmar

M121

Harmonisation ,Innere Medizin“ in Europa

H.S.L.M. Tjen

Studienreise für Ärzte nach Israel

M125

Internationale Kongresse des BDI e.V.

M126

Begrüßung neuer Mitglieder

M127

Weiterbildung

Myokarditis

M. Stille-Siegener, A. Heim, H.-R. Figulla

Medizin aktuell

Leser fragen - Experten antworten

Erratum

Fachnachrichten

766, 774

Buchbesprechungen

780, 787

Kongreßforum

A34

Impressum

A 8

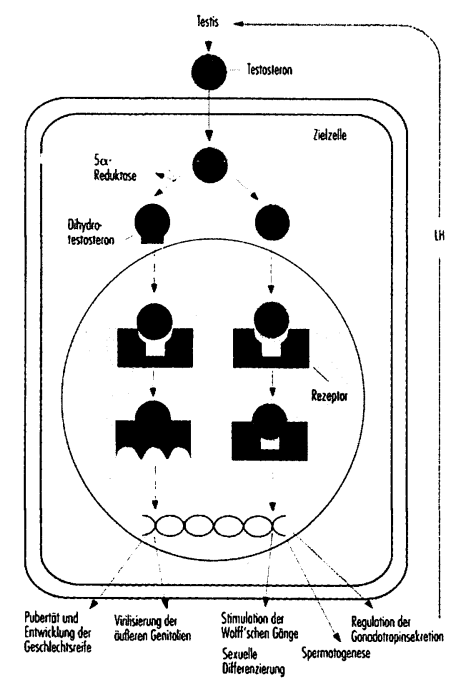

Titelbild: Mechanismus der physiologischen Androgenwirkung. Das im Hoden produzierte Testosteron bindet in den Zielzellen entweder direkt oder nach Umwandlung in Dihydrotestosteron an den Androgenrezeptor.

(Siehe Beitrag T. Gudermann und J. Gromoll, S. 703-711).

Indexed in Current Contents

108 Internist ISSN 0020-9554 INTEAG (1993) 34 (8) 699-804

Gedruckt auf säurefreiem Papier 


\section{Zum Thema}

Die autonome Innvervation des Schwellkörpergewebes erfolgt sympathisch (Th1-L2) und parasympathisch (S2-S4), die somatosensible Versorgung des Penis über die Nn. pudendi (S2-S4). Die Erektion wird durch die Relaxation der glatten kavernösen Muskelzellen bewirkt bei gleichzeitigem Verschlu $\beta$ von arteriovenösen Shunts. Somit füllen sich die Sinusoide arteriell, der venöse Abfluß wird zusätzlich gedrosselt.

Testosteron hat zwar Einflu $\beta$ auf die neuronale Erregungsüberleitung, die für die Erektion wichtig ist, und auf die Libido, nicht aber auf das kavernöse Gewebe. Neurogene Störungen sind nicht selten bei direkter Nervenläsion (z.B. Bandscheibenprolaps, iatrogene Nervendurchtrennung etc.) und diabetischer Neuropathie. Störungen des arteriellen Einstroms sind für ca. $10-15 \%$ erektiler Dysfunktionen verantwortlich, kavernös-venöse Störungen werden bei kavernöser Myopathie beobachtet.

Die Anamneseerhebung, die Untersuchung und die Therapie erektiler Dysfunktionen stellt hohe Ansprüche an das Vertrauensverhältnis von Patient und Untersucher sowie an die ärztliche Gesprächsführung. Als unstrittig kann derzeit gelten, daß organbedingte Erektionsstörungen häufiger vorkommen als früher angenommen wurde.

\section{Schlüsselwörter}

Erektile Dysfunktion - Kavernöse Muskelzelle - Elektromyographie - Stickoxid (NO) - Rekonstruktive Chirurgie

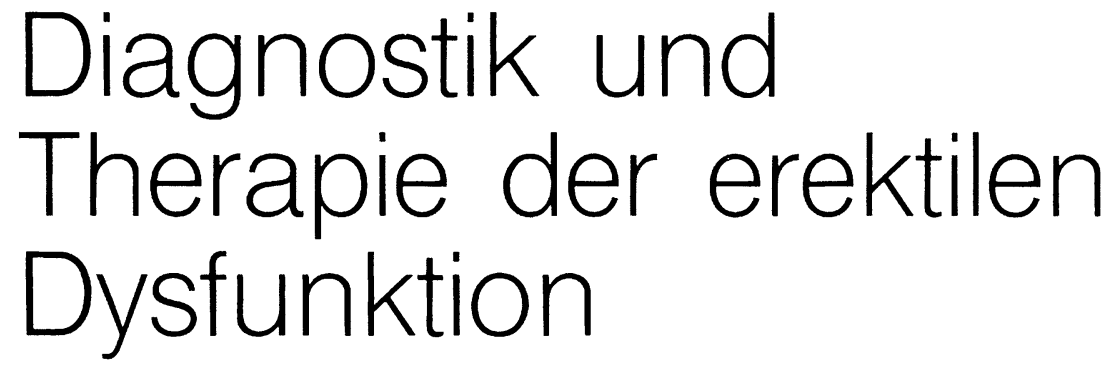

C.G. Stief

Urologische Klinik, Medizinische Hochschule Hannover

Der Diagnostik und Therapie der erektilen Dysfunktion kommt eine große Bedeutung zu; zum einen durch deren hohe Prävalenz, die in Deutschland auf ca. 4 Mio. Männer unter 65 Jahre geschätzt wird, zum anderen durch die erhebliche Belastung des Patienten durch diese spezifische Erkrankung: Durch den Verlust der erektilen Potenz wird das körperliche, seelische und soziale Selbstverständnis des Mannes, insbesondere des jungen Mannes, im Kern erschüttert. Patienten mit chronischer erektiler Dysfunktion sind in ihrem gesamten Verhalten stark verunsichert. Versagens- und Erwartungsängsten kommen bei diesem Phänomen eine mitbedingende und aufrechterhaltende Rolle zu.

Trotz einer Vielzahl von wissenschaftlichen Arbeiten über den Erektionsmechanismus seit Anfang des 16. Jahrhunderts findet sich im medizinischen Schrifttum bis zu Anfang der 70er Jahre unseres Jahrhunderts die Auffassung, daß die erektile Dysfunktion ganz überwiegend psychogen verursacht sei. Diese Auffassung geht auf die Lehrmeinung von Freud zurück, der Erektionsstörungen als Folge von frühkindlichen Entwicklungsstörungen postulierte [5]. Demzufolge wurden bei Patienten mit Erektionsstörungen in über $90 \%$ psychogene Verursachungen diagnostiziert.

Die Erfolge der von Masters und Johnson eingeführten paarorientierten Sexualtherapie sowie die Ent- wicklung der ersten Penisprothesen und die damit erstmals verbundene Behandlungsmöglichkeit von organisch bedingten Erektionsstörungen führten zu einem grundlegenden Wandel in der Auffassung des Krankheitswertes von Erektionsstörungen und deren möglicher Verursachung.

Grundlagenforschung und klinische Studien insbesondere des letzten Jahrzehnts haben zur wesentlichen Erweiterung unseres Wissens über Physiologie und Pathophysiologie des Erektionsvorganges geführt [3, $12,17,22,26]$. Dieses vertiefte Verständnis in den normalen und gestörten Erektionsablauf, die wesentlich verfeinerten und erweiterten diagnostischen Möglichkeiten sowie die multidisziplinäre Abklärung des $\mathrm{Pa}$ tienten ergeben heute ein völlig neues Bild der ursächlichen Entstehung von Erektionsstörungen. Die Komplexität des Erektionsvorganges und die oft multifaktorielle Genese der manifesten Störung erfordern ein fein abgestimmtes Zusammenspiel der verschiedenen Fachdisziplinen.

\section{Anatomie}

Die autonome Innervation des Schwellkörpergewebes erfolgt sympathisch und parasympathisch. Das spinale sympathische Erektionszentrum liegt thorakolumbal in Höhe Th11-L2. Das parasympathische Erektionszentrum ist im Sakralmark in Höhe S2-S4 lokalisiert. Die soma- 
tosensible Innervation des Penis wird über die Nervi pudendi gewährleistet. Der N. pudendus ist aus afferenten und efferenten Anteilen zusammengesetzt, die den Segmenten S2-S4 entspringen [7].

Die arterielle Versorgung der Corpora cavernosa erfolgt durch eine Endarterie der A. iliaca interna, die A. pudenda. Nach Abgabe der A. bulbi urethra zweigt sich die A. pudenda in die A. urethralis, die A. profunda penis und die A. dorsalis penis auf. Der venöse Abstrom der Schwellkörper wird über 3 verschiedene Drainagesysteme gewährleistet.

\section{Physiologie}

Das Phänomen der Erektion wird durch die Relaxation der glatten kavernösen Muskelzellen eingeleitet und unterhalten. Im Vergleich zur Flakzidität wird der arterielle Einstrom zu Beginn der Tumeszenz um das 8 - bis 60 fache gesteigert. Im Gegensatz zum flakziden Stadium, wo der Großteil des arteriellen Blutes über ein Kapillarsystem an den Sinusoiden vorbeigeshuntet wird, erfolgt nun der Verschluß dieser arteriovenösen Shunts und die arterielle Füllung der kavernösen Räume. Parallel mit der Erhöhung des arteriellen Einstroms und der Relaxation der glatten kavernösen Muskulatur geht eine dramatische Drosselung des venösen Abflusses der Schwellkörper einher [12].

In Analogie zum Herz-KreislaufSystem wurde in den letzten Jahren gezeigt, daß der entscheidende Neurotransmitter zur Erektionsinduktion Stickoxid (NO) ist. Peptidergen Substanzen kommt eine modulatorische Bedeutung in der Neurotransmission zu $[1,8,9,18]$.

\section{Pathophysiologie}

\section{Endokrinologische Faktoren}

Die Rolle des Testosterons beim Erektionsvorgang ist noch nicht endgültig geklärt; am kavernösen Gewebe selbst wurden keine signifikanten Mengen von Testosteronrezeptoren gefunden. Es bestehen Hinweise, daß Testosteron einen Einfluß auf die
Empfindlichkeit der für das Erektionsgeschehen wichtigen neuronalen Erregungsüberleitung im zentralen Nervensystem ausübt. Weiterhin ist ein physiologischer Testosteronspiegel für die Libido von entscheidender Bedeutung.

Eine Behandlung von Patienten mit erektiler Dysfunktion und normalem Testosteronspiegel mit Testosteron erbrachte im Vergleich zur Gabe von Placebo keinen signifikanten Therapieerfolg, induzierte aber unerwünschte und potentiell gefährliche hämatogene Veränderungen (Polizythämie). Besteht aber bei einem Patienten ein nachgewiesener Testosteronmangel, sollte dieser entsprechend substituiert werden.

Störungen der erektilen Funktion durch eine Hyperproloaktinämie sind seit längerem bekannt. In einem unselektionierten Patientengut mit erektiler Dysfunktion ist nur bei sehr wenigen Patienten mit einer Erhöhung des Prolaktinspiegels zu rechnen. Nur bei einem Teil der Patienten mit Hyperprolaktinämie, die sich einer medikamentösen Therapie unterzogen, stellte sich die erektile Funktion wieder ein.

Sowohl von Patienten mit hyperthyreoter als auch hypothyreoter Stoffwechsellage wird bei einem groBen Teil von einer Reduktion von Libido und erektiler Potenz berichtet.

\section{Neurogen verursachte Erektionsstörungen}

Sekundäre autonom-neurogene Erektionsstörungen sind häufig anzutreffen. Ätiologisch sind hier in erster Linie internistische Grunderkrankungen, wie z.B. ein Diabetes mellitus, oder Schädigungen der Nerven selbst, z.B. durch (auch subklinische) Bandscheibenvorfälle, zu nennen. Die erektile Dysfunktion nach traumatischer (z.B. durch Beckenringfraktur mit Harnröhrenabriß) oder iatrogener (z.B. durch eine radikale Zystoprostatektomie) Läsion der Nn. erigentes zerstört gleichzeitig den parasympathischen und sympathischen Input des kavernösen Gewebes. Nachdem Studien zu Beginn der 80er Jahre den detaillierten Verlauf dieser feinsten Nerven aufdeckten [24], war eine Entwicklung modifizierter Operationsverfahren mit Schonung der $\mathrm{Nn}$. erigentes möglich.

\section{Kavernöse Myopathie}

Der Tonus der glatten Schwellkörpermuskulatur ist bestimmend für den jeweiligen Funktionszustand des Organs [12]. Degenerative Veränderungen sind demzufolge eng mit Störungen des Erektionsablaufs verbunden [16]. So fanden sich bei mindestens $40 \%$ der Patienten mit venösem Leck in der ultrastrukturellen Untersuchung eine ausgeprägte Degeneration der glatten kavernösen Muskelzellen. Diese myozytäre Degeneration bewirkt eine mangelnde Ausdehnung des kavernösen Gewebes und somit eine ungenügende Tumeszenzzunahme bei der Erektion. Ursächlich beteiligt an dieser Myopathie sind zum einen internistische Leiden wie ein Diabetes mellitus, autonomneurogene Läsionen [16] oder toxische Schädigungen.

\section{Störungen des arteriellen Einstroms}

In großen Multizenterstudien mit multidisziplinärer Abklärung der $\mathrm{Pa}$ tienten wurden bislang bei etwa $40 \%$ der Patienten mit organisch bedingter erektiler Dysfunktion arterielle Einflußstörungen gefunden. Dieser Prozentsatz ist auf Grund neuester kontrollierter Studien als zu hoch anzusehen, da er meist anhand falscher Normalwerte (junge gesunde Patienten anstatt alters- und krankheitsgleiche Kontrollpatienten ohne Störung) berechnet wurde [15]. Heute ist von einem Prozentsatz von ca. 10$15 \%$ arterieller Störungen auszugehen. Morphologisches Substrat dieser Störungen sind entweder angeborene Gefäßdysplasien, traumatische Gefäßabbrüche oder makro- bzw. mikroangiopathische Veränderungen der Gefäße.

\section{Störung der kavernös-venösen Verschlußmechanismen}

Eine mangelnde kavernöse Relaxation führt $\mathrm{zu}$ einer insuffizienten Kompression der subtunikal gelegenen Venenpolster und dadurch zu ei- 
nem vermehrten kavernösen $\mathrm{Ab}$ strom [13, 14]. So ist zu erklären, daß sich bei Patienten mit ausgeprägter kavernöser Myopathie ein venöses Leck diagnostizieren läßt; diese kavernöse Abflußstörung ist chirurgisch-rekonstruktiv nicht therapierbar. Zum anderen besteht bei einem anderen Teil der Patienten eine lokalisierte kavernöse Abflußstörung, welche chirurgisch-rekonstruktiv (,penile Venenchirurgie") angehbar ist.

\section{Diagnostik}

Ziel der Diagnostik der erektilen Dysfunktion ist eine rationelle, aber individuell adaptierte Therapiezuordnung des Patienten. Zur rationellen Abklärung der erektilen Dysfunktion hat sich eine stufenweise Aufteilung der Diagnostik in der Praxis bewährt. Die Basisuntersuchungen werden vom einweisenden Arzt (meist Hausarzt, Internist oder Urologe) durchgeführt. Dann erfolgt die Durchführung des andrologischen nicht- bzw. gering invasiven diagnostischen Programms durch den andrologisch geschulten Urologen. Im Anschluß an diese zweite diagnostische Stufe können die meisten Patienten schon ihrer Therapie zugeführt werden. Nur bei Vorliegen bestimmter Indikationen wird der $\mathrm{Pa}$ tient in dafür eingerichteten Zentren invasiven und aufwendigen, zumeist radiologischen, Untersuchungen (z.B. selektive Phalloarteriographie oder Kavernosometrie und -graphie) zugeführt.

\section{Basisdiagnostik (Stufe I)}

Die wesentlichen Elemente dieser diagnostischen Stufe sind Anamnese, psychologische Evaluierung, körperliche Untersuchung und Labordiagnostik. Wünschenswert ist weiterhin das Ausfüllen eines standardisierten Questionnaires durch den Patienten. Dieses dient zum einen der umfassenden Anamneseerhebung, zum anderen führt es den Patienten in die Vielschichtigkeit der Problematik ein und weckt so Verständnis für die aufwendige Diagnostik.

Neben der Allgemeinanamnese solle das Augenmerk auf Operationen im kleinen Becken, Unfälle, Rükkenmarks- bzw. Wirbelsäulenerkrankungen sowie die klassischen internistischen Risikofaktoren (Nikotinabusus, Diabetes mellitus, Hypercholesterinämie, Makroangiopathie; $[1,17$, 18]) der erektilen Dysfunktion gelegt werden. Situatives (z.B. im Urlaub oder anderen Gelegenheiten mit verringertem Streß) und partnerabhängiges Erektionsverhalten (z.B. mit anderer Partnerin, bei Masturbation) sowie das Auftreten nächtlicher und morgendlicher Erektionen sind zu erfragen.

Die psychologische Evaluierung sollte durch einen in dieser Technik erfahrenen Kollegen durchgeführt werden. Sie ist zu diesem frühen Zeitpunkt in die diagnostische Kaskade zu integrieren, da Patienten mit gravierenden psychogenen Befunden die weitere organische Diagnostik, zumindest vorläufig, erspart werden kann.

An Laborparametern empfiehlt sich routinemäßig die Bestimmung der Elektrolyte, des kleinen Blutbildes, der Blutfette, Nieren- und Leberwerte; an Hormonen genügt als Screening die Bestimmung von Testosteron.

\section{Andrologische nichtinvasive Diagnostik (Stufe II)}

Ziel dieser diagnostischen Stufe ist die Beurteilung der cavernösen Kompetenz, d.h. des Zustands und der funktionellen Kapazität des kavernösen Muskelapparats. Die nicht- bzw. wenig invasiven Methoden SKAT(Schwellkörperautoinjektionstherapie-)Testung und SPACE (,single potential analysis of cavernous electric activity") ermöglichen eine Beurteilung der (funktionellen) penilen Hämodynamik, der penilen autonomen Innervation sowie Rückschlüsse auf den Zustand der kavernösen glatten Muskulatur.

$\mathrm{Zu}$ fast allen Untersuchungen ist anzumerken, daß sie ein funktionelles Organ beurteilen. Eine adäquate Beurteilung setzt eine vollständige Relaxation der glatten kavernösen Muskelzellen voraus. Um die Rate falschpositiver Ergebnisse durch fehlende oder mangelnde Relaxation möglichst niedrig zu halten, muß auf eine entspannte Atmosphäre während der Untersuchungen geachtet werden; Aufregung oder Stre $\beta$ des Patienten gehen mit einer Erhöhung des Sympathikotonus einher, was - noch vor einer Beeinflussung der systemischen Kreislaufparameter - eine Kontraktion der kavernösen glatten Muskeln nach sich zieht [2]. Diese Kontraktion äußert sich dann (trotz ggf. normaler Verhältnisse) z.B. in einer negativen SKAT-Testung, einer pathologischen Dopplerkurve oder einem venösen Leck in der Kavernosometrie.

\section{SKAT-Testung}

Die SKAT-Testung ist eine wenig aufwendige Methode zur globalen Beurteilung der kavernösen Funktionsfähigkeit [20]. Die Erektionsantwort auf die wiederholte, standardisierte intrakavernöse Injektion erlaubt Rückschlüsse auf die penile arterielle Versorgung, den Zustand der glatten kavernösen Muskulatur und die kavernös-venösen Verschlußmechanismen ([20], Abb. 1). Da zum jetzigen Zeitpunkt keine Substanz vom Bundesgesundheitsamt (BGA) zu dieser Indikation zugelassen ist, muß vor dieser Anwendung der Patient ausführlichst aufgeklärt werden (s. unten).

Als geeignete intrakavernöse Injektion hat sich die Kombination aus Papaverin $(15 \mathrm{mg} / \mathrm{ml})$ und Phentolamin $\left(0,5 \mathrm{mg} / \mathrm{ml} ; \quad\right.$ Androskat ${ }^{\circledR}$, Byk Gulden Holland) bewährt; der Zusatz des $\alpha$-Blockers bewirkt zum einen eine wesentliche Wirkungsverstärkung des Papaverins, zum anderen eine Reduktion des (möglichen) Sympathikotonus durch die Blockade der $\alpha$-Rezeptoren des Schwellkörpergewebes [26]. Zur Sicherung der Diagnose sollten mindestens 3 Injektionen (höchstens eine Injektion pro Tag!) durchgeführt werden. Die größte Gefahr bei der SKAT-Testung stellen die prolongierten Erektionen $(>6 \mathrm{~h})$ dar, die in bis zu $10 \%$ der Patienten auftreten können [20]. Die Möglichkeit der Behandlung dieser Komplikation ist unabdingbare Voraussetzung der SKAT-Testung. 


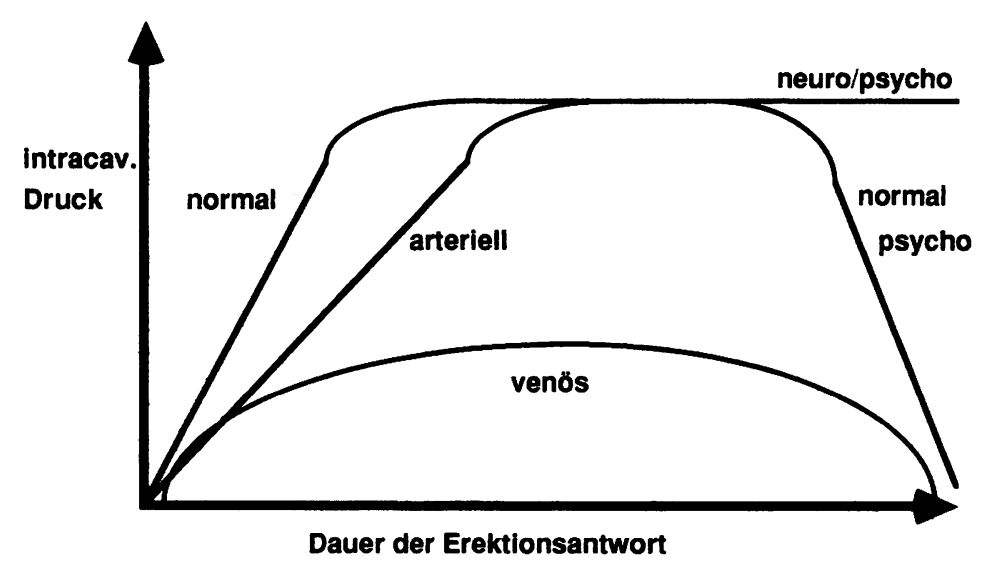

Abb. 1. Abhängigkeit der Erektionsantwort auf eine standardisierte intrakavernöse Injektion von der Ätiologie der erektilen Dysfunktion

Die gravierende Komplikation „prolongierte Erektion“ bei der Verwendung von Papaverin-Phentolamin sowie die erhebliche Schmerzrate $(20-30 \%)$ bei der Applikation von Prostaglandin E1 (PGE1) während der diagnostischen Anwendung legen den Ersatz dieser Substanzen durch den NO-Donor SIN 1 (Corvasal Intracoronaire ${ }^{\circledR}$, Höchst Frankreich) nahe [22].

\section{SPACE}

Während das EMG der quergestreiften Muskulatur seit Jahrzehnten eine zentrale Rolle in der (neuro)myogenen Diagnostik innehat, konnte das EMG glatter Muskelzellverbände keine größere klinische Bedeutung erlangen. Dies war zum einen auf die noch nicht in ihrer Bedeutung erkannten autonomen Funktionsstö- rungen, zum anderen auf die schlechte Zugänglichkeit der Organe (Magen, Darm, Harnleiter) zurückzuführen. Die Ableitung des penile-kavernösen EMG (SPACE) erlaubt nun zum ersten Mal eine elektromyographische Beurteilung eines glattmuskulären Organes in der klinischen Routine [6, 21].

Die SPACE-Untersuchung (,single potential analysis of cavernous electric activity") dient der Registrierung der kavernösen elektrischen Aktivität. Normalerweise läßt diese im flakziden Zustand bestimmte Muster erkennen: Phasen ausgeprägter elektrischer Aktivität, sog. „Potentiale“ von 12-18 s Dauer und einer Frequenz von ca. $0,4-2,5 / \mathrm{min}$, werden von Phasen elektrischer Ruhe gefolgt (Abb. 2). Bei Patienten mit neurologisch definierten Läsionen oder einer

kavernösen Myopathie zeigen sich spezifische Änderungen dieser Erregungsmuster (Abb. 3). In jüngster Zeit entwickelten wir computergestützte Analyseprogramme zur Auswertung dieser elektrischen Aktivitätsmuster, die die Interpretation wesentlich erleichtern. Sowohl in Form der analogen Registrierung als auch der computergestützten Aufarbeitung ermöglicht SPACE die Diagnose von neurogen-autonom und/oder kavernös-myopathisch bedingten Erektionsstörungen, was eine entscheidende therapierelevante Bedeutung besitzt.

Die folgenden beiden Untersuchungen sind noch der Stufe II zuzurechnen, können aber bei einem Teil der Patienten entfallen (s. Abb. 4):

\section{Doppler-Sonographie}

Die Doppler-Sonographie dient der Beurteilung der funktionellen Kapazität der penilen Arterien [13]. Im flakziden Zustand wird ein großer Teil des arteriellen kavernösen Blutes mittels Shunts an den Schwellkörperkavernen vorbeigeführt. Nur im Stadium der Tumeszenz kommt es zu einem maximalen Einstrom in die kavernösen Sinus bei gleichzeitigem Verschluß der a.v.-Shunts. Aus diesem Grunde ist die Doppler-Untersuchung der penilen Gefäße nach intrakavernöser Injektion von SKAT nicht nur wesentlich vereinfacht, sondern überhaupt erst aussagekräftig.

\section{Registrierung der penilen}

Tumeszenz und Rigidität

Die klassische Untersuchungsmethode als Differenzierungshilfe zwischen psychogener und organogener Erektionsstörung ist die Registrierung der

Abb. 2. SPACE-Befund bei einer 34jährigen Normalperson. Die elektrische Aktivität ist in beiden Corpora cavernosa synchronisiert (oben rechtes C. cavernosum, unten linkes), die Potentiale zeigen eine normale Form. Es fehlen pathologische Potentiale. Die horizontale Markierung stellen $5 \mathrm{~s}$, die vertikale $100 \mu \mathrm{V}$ dar

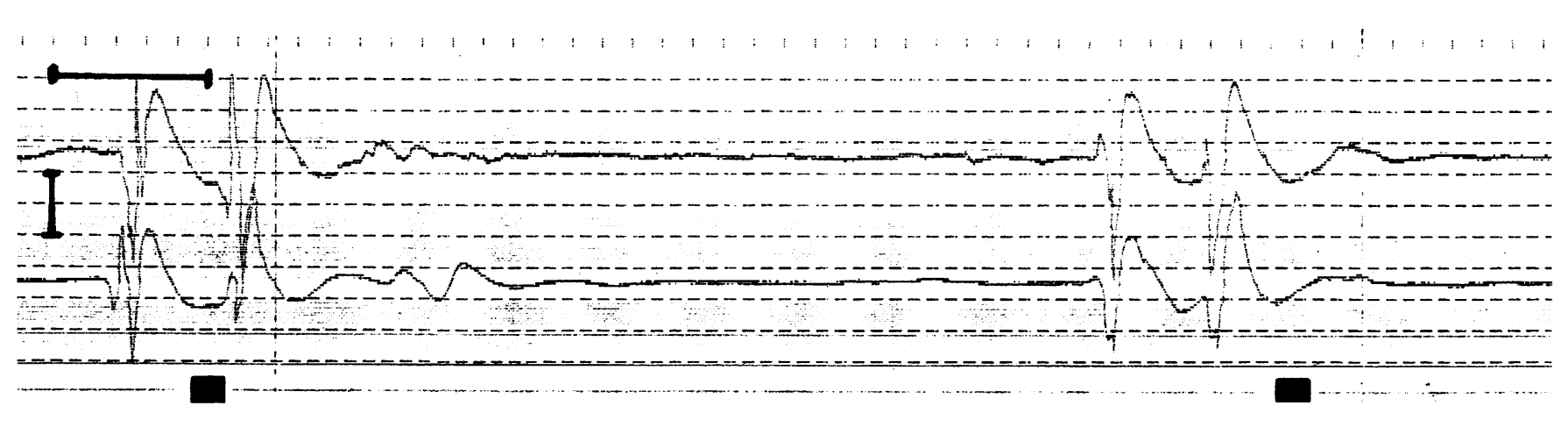




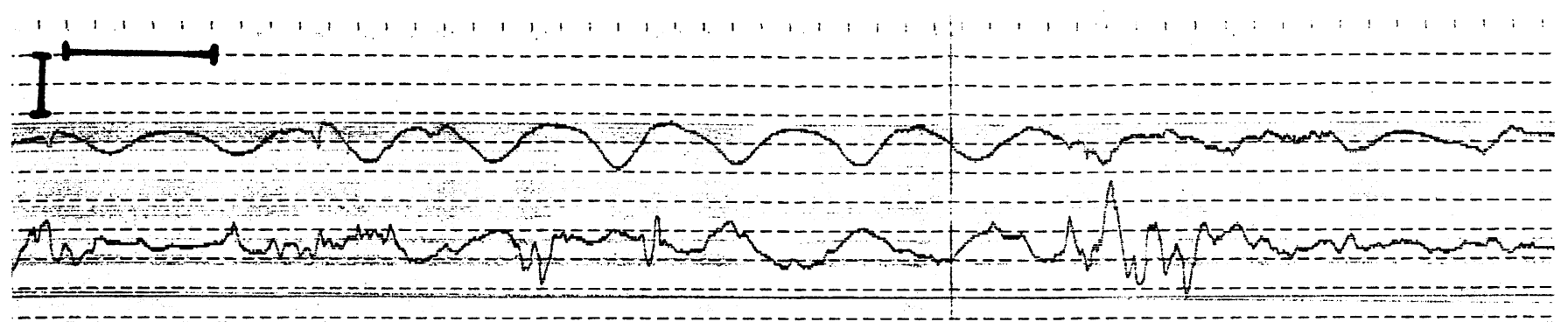

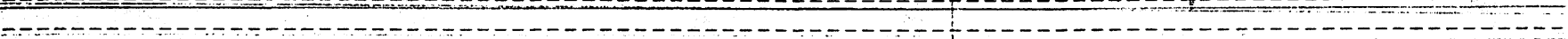

Abb. 3. SPACE-Befund nach radikaler Zystoprostatektomie mit konsekutiver peripherer autonomer Denervierung. Die elektrische Aktivität zeigt keine Synchronisation mehr, die Potentialformen weichen deutlich von der normaler Potentiale (s. Abb. 2) ab. Die horizontale Markierung stellen $5 \mathrm{~s}$, die vertikale $100 \mu \mathrm{V}$ dar

Anamnese, körperliche Untersuchung, Labor, psychologisches

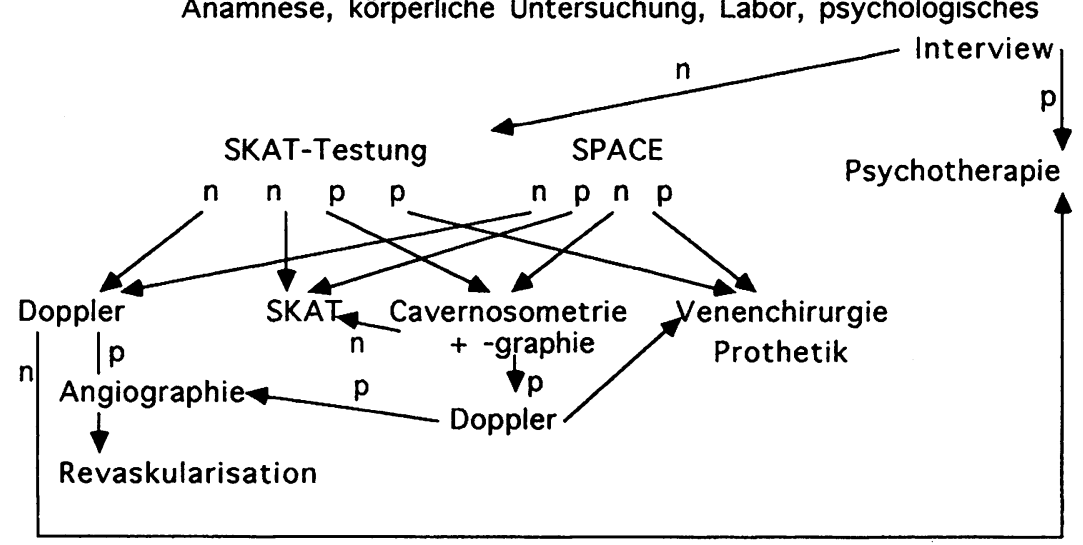

Abb. 4. Algorithmus der Diagnostik und Therapie der erektilen Dysfunktion ( $n$ Normalbefund, $p$ pathologisch)

penilen Tumeszenz und Rigidität während des nächtlichen Schlafs (,NPT-Messung"). Neuere Forschungsergebnisse [7] lassen jedoch erhebliche Zweifel an der Folgerung „nächtliche Erektion = Erektion bei sexueller Erregung" und damit an der Grundvoraussetzung dieser Methode aufkommen.

Deswegen wird heute als Screeningmethode die Registrierung der penilen Tumeszenz und Rigidität während AVSS vor und nach intrakavernöser Injektion vasoaktiver Substanzen $(0,2 \mathrm{ml} \mathrm{SKAT})$ durchgeführt. Größere Studien der jüngeren Zeit belegten eine Bedeutung dieser Methode bei der Differenzierung in psychogene oder organogene Verursachung der Erektionsstörung.

Nach Abschluß der zweiten diagnostischen Stufe ist eine therapierelevante Zuordnung des überwiegen- den Teils (ungefähr 60-80\%) der Paweitere Untersuchungen notwendig sein, so sollte der Patient an eine spezialisierte Institution überwiesen werden.

\section{Invasive andrologische Diagnostik} (Stufe III)

Die invasive andrologische Diagnostik besteht im wesentlichen aus der selektiven Pharmakophalloarteriographie (radiologische Darstellung des penilen Einstroms) sowie der Pharmakokavernosometrie und -kavernosographie (Quantifizierung und Darstellung des kavernösen Abstroms, [14]). Diese Untersuchungsverfahren dienen der Vorbereitung der operativen rekonstruktiven Maßnahmen. Auf Grund ihrer Invasivität sollten sie nur bei - im positiven Falle tienten möglich (s. Abb. 4). Sollten
- Bereitschaft des Patienten zur operativen Therapie durchgeführt werden.

\section{Therapie von Erektionsstörungen}

Zur Behandlung von organischen Erektionsstörungen stehen dem Therapeuten heute die orale Medikation, die intrakavernöse Injektionstherapie (SKAT), die penile Venenchirurgie, die arterielle Revaskularisation sowie die prothetische Versorgung zur Verfügung (einen möglichen Algorithmus zeigt das Flußdiagramm in Abb. 4). Die Akzeptanz von Vakuumpumpen ist (in unserem Krankengut) gering.

Bei Patienten mit rein oder überwiegend psychogener Verursachung sollte die Therapie von einem geschulten Psychologen oder Psychiater geleitet und überwacht werden.

Neben den klassischen (und zeitlich aufwendigen) Optionen wie $\mathrm{Ge}$ sprächs- oder Paartherapie kann insbesondere bei Versagens- und/ oder Erwartungsangst ein Therapieversuch mit oraler Medikation, wie z.B. Yohimbin (Yohimbin Spiegel ${ }^{\circledR}$ ), 3 mal $5 \mathrm{mg}$ für 3 Tage, dann $3 \mathrm{mal}$ $10 \mathrm{mg}$, unternommen werden. Vor einer endgültigen Bewertung eines möglichen Therapieerfolges sollte eine Mindesteinnahmedauer von 8 Wochen abgewartet werden. Ging man bis vor kurzem noch von einer sehr geringen therapeutischen Effizienz dieser Substanz aus, so konnten neuere Untersuchungen zeigen, $\mathrm{da} \beta$ bei geeigneter Selektion der $\mathrm{Pa}$ tienten beachtliche Behandlungserfolge $(>30 \%) \mathrm{zu}$ erzielen sind [8]. Dieser Therapieversuch bietet sich auch bei Patienten mit unauffälligen psychogenen Befunden und lediglich subsignifikanten organogenen Ergebnissen an, da die beobachteten Ne- 
benwirkungen von geringfügiger $\mathrm{Na}$ tur waren.

Standardtherapie bei Patienten mit organogener erektiler Dysfunktion, bei denen eine rekonstruktive Operation nicht aussichtsreich erscheint oder nicht gewünscht wird, ist die Schwellkörperautoinjektionstherapie (SKAT). Hierbei wird mit einer Insulinnadel $(26$ gg. $\times 1 / 2)$ der Schwellkörper einseitig von lateral (oder dorsal) punktiert und das Substanzgemisch ohne vorherige Aspiration appliziert. Zur Vermeidung bzw. Reduktion möglicher Nebenwirkungen sollten Patienten mit schweren Herzrhythmusstörungen, AVK > III, schweren Leber- oder Nierenschäden, Suchterkrankungen und ausgeprägter Deviation von der SKAT ausgeschlossen werden. Unbedingte Voraussetzung zur SKAT sind ein kooperationsfähiger Patient und ein ständig erreichbarer Therapeut.

Nach Erlernen der Autoinjektionstechnik und Adaptation der Dosis (angestrebte Dauer der Erektion ca. 30-60 min) wird der Patient in die häusliche Applikation entlassen. Da unter häuslichen Bedingungen durch die zusätzliche Stimulation eine erhebliche Dosisverstärkung zu erwarten ist, wird die Dosierung um ca. 1/3 im Vergleich zur Testdosis gesenkt. Der Patient sollte höchstens 2-3 Injektionen pro Woche durchführen; zur Vermeidung prolongierter Erektionen ist, auch im Falle eines Nichterfolgs, eine Nachinjektion am gleichen Tage zu verbieten. Beim Auftreten der gefährlichsten Sofortnebenwirkung, der prolongierten Erektion (Dauer über 4 h) mit der Gefahr der irreversiblen kavernösen Schädigung, muß sich der Patient zur Einleitung von geeigneten Maßnahmen sofort bei seinem behandelnden Arzt, oder einem in dieser Therapie Erfahrenen, einfinden.

Zur frühzeitigen Erkennung lokaler oder systemischer Nebenwirkungen ist eine engmaschige Nachkontrolle (zuerst nach 10, dann nach je 25 Autoinjektionen) des Patienten mit Anamnese, körperlicher Untersuchung und Labor erforderlich. Im Falle des Auftretens von kavernösen Fibrosen muß sofort eine Injektions- pause von 2-3 Monaten eingelegt werden. Sollten sich die Fibrosen in diesem Zeitraum zurückgebildet haben, kann, nach ausführlicher Abwägung, eine erneute SKAT mit einer anderen Substanz versucht werden [11].

Zum jetzigen Zeitpunkt ist beim BGA noch keine Substanz zur intrakavernösen Applikation mit dieser Indikationsstellung zugelassen. Aus diesem Grund stellt diese Therapie einen Heilversuch dar, wobei jeder Patient über Risiken und mögliche Nebenwirkungen (insbesondere Nichtzulassung der Substanz, prolongierte Erektion, Fibrose, vollständige Impotenz, Kreislaufversagen, Schock, Krebsentstehung) ausführlich aufgeklärt werden muß; diese Aufklärung ist $\mathrm{zu}$ dokumentieren und vom $\mathrm{Pa}$ tienten zu unterschreiben.

Auf Grund wissenschaftlicher Untersuchungen und klinischer Erfahrungen ist zum heutigen Zeitpunkt von der intrakavernösen Injektion von Papaverin als Monosubstanz abzuraten. Es hat sich hier gezeigt, da $\beta$ im Vergleich zu anderen Substanzen zum einen die Ansprechrate in bezug auf das Erreichen einer vollen Erektion deutlich geringer, zum anderen das Auftreten von intrakavernösen Fibrosen signifikant häufiger ist. Eher zu empfehlen ist zum jetzigen Zeitpunkt die Verwendung einer Mischung von Papaverin $(15 \mathrm{mg} / \mathrm{ml})$ und Phentolamin $(0,5 \mathrm{mg} / \mathrm{ml}$, Androskat ${ }^{\circledR} /$ Byk Holland) in einer Dosierung von bis zu $2 \mathrm{ml}$ dieses Gemisches oder die Applikation von Prostaglandin E1 bis zu einer Dosierung von $20 \mu \mathrm{g}$ [11].

Neueste wissenschaftliche Ergebnisse lassen annehmen, daß der Stickoxid(NO)-Donor SIN-1 (Corvasal Intracoronaire ${ }^{\circledR}$, Höchst Frankreich) die Standardsubstanz der SKAT werden könnte [22]. Aufgrund der Eigenschaften dieser Substanz scheint die Gefahr der prolongierten Erektion gebannt (bislang wurde, allerdings in einer relativ kleinen Serie von weniger als 200 Patienten, noch keine prolongierte Erektion beobachtet). Weiterhin scheint SIN-1 sehr gut gewebeverträglich zu sein, was sich in der Abwesenheit von Schmerzsensationen bei der Injektion selbst sowie erfolgversprechender lokaler Toxizi- tätsstudien verschiedener Tierspezies bestätigte.

Treten bei der Kombination von Papaverin und Phentolamin im Vergleich zu PGE1 in einem höheren Maße prolongierte Erektionen auf, so ist bei der Verwendung von PGE1 bei etwa $20-30 \%$ (!) der Patienten mit z.T. sehr ausgeprägten Schmerzen bei der Erektion zu rechnen. SIN-1 scheint keine dieser gravierenden $\mathrm{Ne}-$ benwirkungen der beiden vorgenannten Substanzen zu besitzen.

Jüngeren Patienten mit rein arterieller Genese der erektilen Dysfunktion (evtl. mit gering ausgeprägtem venösen Leck) kann eine arterielle Revaskularisation angeboten werden. Hierbei wird mit mikrochirurgischen Techniken eine Anastomose zwischen a. epigastrica inferior und a. dorsalis penis (evtl. mit gleichzeitigem Shunt zur v. dorsalis penis) angelegt [18]. Wurde dieses Verfahren noch vor wenigen Jahren häufig empfohlen und auch durchgeführt, so mahnen die postoperativen Ergebnisse doch zu großer Zurückhaltung gegenüber dieser Therapieoption. Hier stehen einer geringen Erfolgsrate (0-40\%) eine doch erhebliche Morbidität des Eingriffs (bis 30\% Glanshyperämien mit der Gefahr der Glans- oder Harnröhrennekrose) entgegen.

Patienten mit rein venöser Genese kann zur Reduktion des pathologischen kavernös-venösen Abstroms eine penile Venenligatur vorgeschlagen werden; nach kavernosographischer Dokumentation werden die dorsalen oberflächlichen Venen ligiert bzw. die tiefe dorsale Vene reseziert [23]. Dieser Eingriff besitzt eine sehr geringe Morbidität. Auch bei diesem grundsätzlich attraktiven chirurgischen Verfahren ist anzumerken, daß über die Indikationsstellung keine allgemeine Übereinstimmung herrscht und bezüglich der Langzeitergebnisse eher entmutigende Daten vorliegen. Neueste Ergebnisse legen aber nahe, da $\beta$ der SPACE-Untersuchung eine entscheidende Bedeutung in der präoperativen Selektion zukommt und damit die postoperativen Ergebnisse wesentlich verbessert werden können.

Obwohl die am längsten verfügbare therapeutische Option bei organischen Erektionsstörungen, so kommt 
der prothetischen Versorgung heute ein Platz entweder nur noch als ultima ratio oder auf Wunsch des Patienten zu. Es ist aber gleichfalls festzustellen, daß bei richtiger Indikationsstellung sowie Aufklärung von $\mathrm{Pa}$ tient und Partnerin über die Limitationen dieser Therapieform die alloplastische Versorgung mit einer hohen Akzeptanz und Zufriedenheit der Patienten verbunden ist. Grundsätz-

\section{Fazit für die Praxis}

Ist eine Erektionsstörung überwiegend psychogen bedingt, bedarf der Patient kompetenter psychologischer, psychiatrischer oder psychotherapeutischer Hilfestellung. Diese kann besonders bei Versagensund/oder Erwartungsangst durch medikamentöse orale Gabe von Johimbin unterstützt werden. Dies sollte über mindestens zwei Monate eingenommen werden. Bei geeigneter Selektion liegen die Behandlungserfolge $>30 \%$.

Bei organogener erektiler Dysfunktion gilt derzeit die Schwellkörperautoinjektionstherapie (SKAT) als Therapiemethode der Wahl. Mittels Insulinnadel erfolgt die Injektion in den Schwellkörper und bewirkt eine Erektion von 30 bis 60 Minuten. Durch neuentwickelte Substanzen wird die Gefahr einer unerwünscht prolongierten Erektion minimiert.

Liegt bei jüngeren Patienten eine rein arterielle Genese vor, kann eine arterielle Revaskularisation erwogen werden, die allerdings mit gravierenden Komplikationen verbunden sein kann. Bei venöser Genese kann eine penile Venenligatur vorgenommen werden, über deren Indikation und Langzeiterfolge es allerdings divergierende Meinungen gibt.

Die prothetische Versorgung gilt als ultima ratio, sie führt aber bei Akzeptanz durch beide Partner auch zu deren Zufriedenheit, wobei aufblasbare Modelle zu besseren kosmetischen Ergebnissen als semirigide Prothesen führen. lich stehen eine Vielzahl von semirigiden und aufblasbaren Prothesen zur Verfügung, wobei das bessere kosmetische Ergebnis der aufblasbaren Modelle im Vergleich zu den semirigiden auch nach wesentlichen Verbesserungen durch eine erhebliche Reparaturanfälligkeit erkauft wird.

\section{Zusammenfassung}

Störungen der Erektionsfähigkeit des Mannes haben eine erhebliche Auswirkung auf die physische und psychische Verfassung des Patienten und sind als ernstzunehmende Krankheit einzustufen. Sowohl beim Zustandekommen einer Erektion als auch in der Verursachung einer erektilen Dysfunktion kommt der kavernösen glatten Muskulatur eine Schlüsselrolle zu. Die Diagnostik dieses ätiologisch multifaktoriellen Krankheitsbildes erfordert eine interdisziplinäre Abklärung, was durch eine stufenweise, rationalisierte Organisation erleichtert wird. Die Standardbehandlung der organisch verursachten Erektionsstörung stellt die Schwellkörperautoinjektionstherapie dar. Hier scheint der Einsatz von Substanzen, die den physiologischen Erektionsmechanismus imitieren (NO-Donoren), im Vergleich zu den bislang üblichen Medikationen eine deutlich nebenwirkungsärmere Therapie zu gestatten.

\section{Literatur}

1. Azadzodi KM, de Tejada IS (1991) Hypercholesterolemia impairs endothelium dependent relaxation of rabbit corpus cavernosum smooth muscle. J Urol 145:238-243

2. Benard F, Diederichs W, Stief CG, Bosch $\mathrm{R}$, Aboseif S, Lue TF, Tanagho EA (1989) The effect of epinephrine on erection. J Urol 141:439A

3. Brindley GS (1983) Cavernosal alphablockade: a new technique for investigating and treating erectile impotence. $\mathrm{Br} \mathrm{J}$ Psychiatry 143:332-337

4. Ebbehoj J, Wagner G (1979) Insufficient penile erection due to abnormal drainage of cavernous bodies. Urology 13:507510

5. Freud S (1964) Vorlesungen zur Einführung in die Psychoanalyse. Fischer, Frankfurt

6. Gerstenberg TC, Nordling J, Hald H, Wagner G (1989) Standardized evalu- ation of erectile dysfunction in 95 consecutive patients. J Urol 141:857-863

7. de Groat WC, Steers WD (1988) Neuroanatomy and neurophysiology of penile erection (1988) In: Tanagho EA (ed) Contemporary management of impotence and infertility. Williams \& Wilkins, Baltimore

8. Hartmann U, Stief C, Djamilian M, Thon W, Fritz KW, Langer D, Allhoff EP, Jonas U (1991) Therapieversuch der erektilen Dysfunktion mit oraler Medikation bei selektionierten Patienten. Urologe [B] 31:204-207

9. Holmquist F, Stief CG, Jonas U, Andersson K-E (1991) Effects of the nitric oxide synthase inhibitor $\mathrm{N}^{\mathrm{G}}$-nitro-L-arginine on the erectile response to cavernous nerve stimulation in the rabbit. Acta Physiol Scand 143:299-305

10. Ignarro JL, Bush PA, Buga GM, Wood KS, Fukoto JM, Raifer J (1990) Nitric oxide and cyclic GMP formation upon electrical field stimulation cause relaxation of corpus cavernosum smooth muscle. Biochem Biophys Res Commun 170:843-854

11. Jünemann KP, Alken P (1989) Pharmacotherapy of erectile dysfunction (1989). Int $J$ Impotence Res 1:71

12. Lue TF, Takamura T, Schmid RA, Tanagho EA (1983) Hemodynamics of erection in the monkey. J Urol 130:12311237

13. Lue TF, Hricak H, Marich KW, Tanagho ET (1985) Evaluation of arteriogenic impotence with intracorporal injection of papaverine and the duplex ultrasound scanner. Semin Urol 3:43-49

14. Lue TF, Hricak H, Schmidt RA, Tanagho EA (1986) Functional evaluation of penile veins by cavernosography in papaverine-induced erection. $\mathrm{J}$ Urol 135:479-484

15. Meuleman EJH, Belemans BLH, Doesburg WH, Asten WNJC, Skotnicki SH, Debruyne FMJ (1992) Penile pharmacological duplex ultrasonography: a dose effect study comparing papaverine, papaverine/phentolamine and prostaglandine E1. J Urol 148:63

16. Persson C, Diederichs W, Lue TF, Yen TS, Fishman I, McLin PH, Tanagho ET (1989) Correlation of altered ultrastructure with clinical arterial evaluation. $\mathrm{J}$ Urol 142: 1462-1469

17. de Tejada S, Goldstein I, Krane RJ (1988) Local control of penile erection. Urol Clin North Am 15:9-15

18. de Tejada IS, Goldstein I, Azadzodi K, Krane R, Cohen R (1989) Impaired neurogenic and endothelium mediated relaxation of penile smooth muscle from diabetic men with impotence. $\mathrm{N}$ Engl J Med 320:1025-1031

19. Sohn M, Bohndortf K, Sikora R, Albrecht D, Jakse G (1992) Selective dyna- 
mic pharmaco-angiography of penile vessels: correlation to intraoperative findings. Z Urol (Poster) 4:50-53

20. Stief CG, Bähren W, Gall H, Scherb W (1988) Functional evaluation of penile hemodynamics. J Urol 139:734

21. Stief CG, Djamilian M, Schaebsdau F, Truss M, Abicht J, Allhoff EP, Jonas U (1990) Single potential analysis of cavernous electric activity. World J Urol 8:7583

22. Stief C, Holmquist F, Djamilian M, Krah H, Andersson KE, Jonas U (1992) Preliminary results with the nitric oxide donor linsidomine cholalhydrate in the treatment of human erectile dysfunction. $\mathrm{J}$ Urol 148:1437-1440

23. Virag R (1982) Intracavernous injection of papaverine for erectile failure. Letter to the editor. Lancet 2:938

24. Walsh PC, Donker P (1982) Impotence following radical retropubic prostatectomy. J Urol 128:492

25. Wespes E, Schulman CC (1985) Surgical treatment of a curable cause of impotence. J Urol 133:796-769

26. Zorgniotti AW, Lefleur RS (1985) Autoinjection of the corpus cavernosum with a vasoactive drug combination for vasculogenic impotence. J Urol 133:39-42

Priv.-Doz. Dr. C.G. Stief

Urologische Klinik

Medizinische Hochschule Hannover

D-30623 Hannover 